\title{
Research on 3D Simulation System and Algorithm for Robot Teleoperation
}

\author{
LinlinYan ${ }^{1,2,3}$, FangXu $^{1,3}$, KaiJia ${ }^{1,3}$ \\ ${ }^{1}$ Shenyang Institute of Automation, Chinese Academy of Sciences, Shenyang, 110016, China \\ ${ }^{2}$ University of Chinese Academy of Sciences, Beijing, 100049, China \\ ${ }^{3}$ SIASUN Robot \&Automation Co., Ltd, Shenyang 110168, China
}

Keyword: robot teleoperation; kinematics algorithm; master-slave control; 3D dynamic simulation

\begin{abstract}
For experiment platform of robot teleoperation control system requires expensive robot and master equipment, a method of robot construction of 3D simulation system for robot teleoperation is presented. According to the analysis of composition of robot teleoperation control system, the master equipment of Delta parallel mechanism is chosen, Delta parallel robot and SCARA serial robot is chosen as the slave-robot, and TCP/IP protocol network is chosen as communication model between the master and the slave. According the study of the kinematics algorithm for the master and the slave for robot teleoperation system, and the study of control algorithm for robot teleoperation system, the 3D dynamic simulation system for robot teleoperation control system is developed by use of the mixed programming of MFC and OpenGL. The practical simulations verify the reliability of the control algorithms of robot teleoperation control and the communication model, and show the value of the simulation system of teleoperation in the research for the robot teleoperation control.
\end{abstract}

\section{Introduction}

Teleoperation system is a complex control system, by which the remote device can complete the complex operation in the environment away from the operator. Robot teleoperation as an indispensable robot control method in the process of roboth as been studied and a keen height the opinion by many experts and scholars[1-2].Robot teleoperation control system platform needs some expensive equipment such as the master equipment and the slave robot, people have to give up the research on Robot teleoperation control system because of funding problems in some cases.Therefore, the developmentof3D robot teleoperation control simulation platform has important significance for the study of control algorithm in the robot research field[3].

The master as the interaction equipment by which human control the remote robot,and the force feedback is often added to it to feel the movement states of the robot.The characteristic of parallel mechanism determines the force feedback can be added to it better [4], and Delta as a very successful parallel mechanism is trusted deeply by the researchers[5].Robot teleoperation control includes mainly the master-slave isomorphism control and the master-slave isomeric control according to the different mechanism, so that the research on isomorphism control and isomeric control is another important problem. Meanwhile, OpenGL as a kind of excellent graphical programming interface, it has strong ability of construction of 3D graphics, and far more excellentin graphics performance than MATLAB that often be used in the study control algorithm, and which has been widely favoured in robot simulation fields [6]

In this paper,the 3D model of Delta parallel master, isomorphism Delta slave robot and isomeric

SCARA slave robot, the master simulation platform and the slave simulation platform are designed, the communication system between the master and the slave is built, and the verification for the reliability of the control algorithms and stability of the simulation system is conducted in experiments. 


\section{The kinematic and 3d simulation of the master-mechanism}

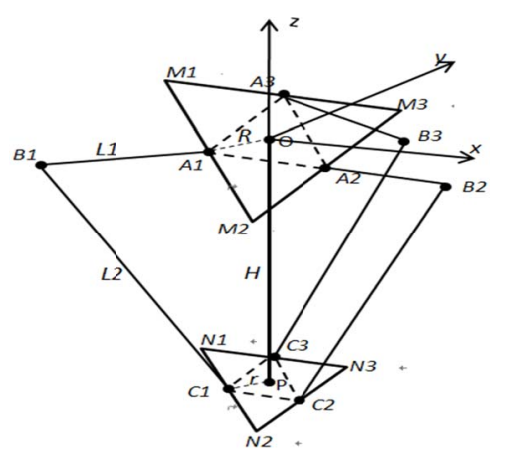

Fig.1:The structure of kinematics modeling for Delta parallel mechanism

As can be seen from Fig.1, the static platform of parallel mechanism is constituted of point ${ }^{M_{1}}$, $M_{2}$ and ${ }^{M_{3}}$, and the moving platform is constituted of points ${ }^{N_{1}},{ }^{N_{2}}$ and ${ }^{N_{3}}$. Points ${ }^{A_{1}}, A_{2}$ and ${ }^{A_{3}}$ are the middle point of the three sides of the static platform, and Points ${ }_{1}, C_{2}$ and $C_{3}$ are the middle point of the three sides of the static platform. $\mathrm{R}$ and $\mathrm{r}$ are the radius of inscribed circle of the static platform and the moving platform, ${ }^{L_{1}}$ and ${ }^{L_{2}}$ are the length of master rod and slave rod, $\mathrm{H}$ is the length in the two middle points of the static platform and the moving platform. According to the movement characteristics of the mechanism, the base coordinate system is placed in the center of the static platform.

\subsection{Inverse kinematics analysis of the master-mechanism}

The kinematics of parallel mechanism can't be analysed by traditional d-h method, so $\mathrm{x}, \mathrm{y}$ and $\mathrm{z}$ is the end coordinates of the master-mechanism, ${ }_{1}, \theta_{2}$ and $\theta_{3}$ are the three driving rod rotation angles, ${ }^{\partial_{1}}$, $\partial_{2}$ and $\partial_{3}$ are the angles from axis-x to the $\overrightarrow{O A_{1}}, \overrightarrow{O A_{2}}$ and $\overrightarrow{O A_{3}}$. As can be seen from Fig. $1, \partial_{1}=210^{\circ}$ 、 $\partial_{2}=330^{\circ} 、 \partial_{3}=90^{\circ}$ 。

According to the first drive branched chain relationship with the mechanism, the following formula is gotten:

$$
\begin{gathered}
\overrightarrow{B_{1} C_{1}}=\overrightarrow{O P}-\overrightarrow{O A_{1}}-\overrightarrow{A_{1} B_{1}}-\overrightarrow{C_{1} P} \\
\overrightarrow{O P}=\left[\begin{array}{l}
\mathrm{x} \\
y \\
z
\end{array}\right], \quad \overrightarrow{O A_{2}}=\left[\begin{array}{ccc}
\cos \partial_{2} & -\sin \partial_{2} & 0 \\
\sin \partial_{2} & \cos \partial_{2} & 0 \\
0 & 0 & 1
\end{array}\right]\left[\begin{array}{l}
R \\
0 \\
0
\end{array}\right], \\
\overrightarrow{A_{1} B_{1}}=\left[\begin{array}{ccc}
\cos \partial_{1} & -\sin \partial_{1} & 0 \\
\sin \partial_{1} & \cos \partial_{1} & 0 \\
0 & 0 & 1
\end{array}\right]\left[\begin{array}{l}
L_{1} \cos \partial_{1} \\
0 \\
L_{1} \sin \partial_{1}
\end{array}\right], \\
\overrightarrow{C_{1} P}=\left[\begin{array}{ccc}
\cos \partial_{1} & -\sin \partial_{1} & 0 \\
\sin \partial_{1} & \cos \partial_{1} & 0 \\
0 & 0 & 1
\end{array}\right]\left[\begin{array}{l}
-\mathrm{r} \\
0 \\
0
\end{array}\right],
\end{gathered}
$$

Then from formula (1), formula(2)can be gotten

$$
\left|\overrightarrow{B_{1} C_{1}}=\overrightarrow{O P}-\overrightarrow{O A_{1}}-\overrightarrow{A_{1} B_{1}}-\overrightarrow{C_{1} P}\right|
$$

Then from formula (2), formula(3)can be gotten

$$
\begin{aligned}
& {\left[\left(\mathrm{x}+\mathrm{r} \cos \partial_{1}-R \cos \partial_{1}\right)-\mathrm{L}_{1} \cos \partial_{1} \cos \theta_{1}\right]^{2}+\left[\left(\mathrm{y}+\mathrm{r} \sin \partial_{1}-R \sin \partial_{1}\right)-\mathrm{L}_{1} \sin \partial_{1} \sin \theta_{1}\right]^{2}+} \\
& {\left[z-\mathrm{L}_{1} \sin \theta_{1}\right]^{2}-L_{2}^{2}=0}
\end{aligned}
$$

Next:

$$
\begin{gathered}
\mathrm{u}_{1}=x+r \cos \partial_{1}-R \cos \partial_{1}, \\
\mathrm{v}_{1}=y+r \sin \partial_{1}-R \sin \partial_{1}, \\
\mathrm{~m}_{1}=-2 u_{1} L_{1} \cos \partial_{1}-2 v_{1} L_{1} \sin \partial_{1}, \\
\mathrm{n}_{1}=-2 z L_{1},
\end{gathered}
$$


Then formula(4)can be gotten

$$
p_{1}=u_{1}^{2}+v_{1}^{2}+z^{2}+L_{1}^{2}-L_{2}^{2}
$$

$$
\mathrm{m}_{1} \cos \theta_{1}+n_{1} \sin \theta_{1}+p_{1}=0
$$

Then $\theta_{1}$ can be gottenFrom formula (4). Similarly, $\theta_{2}$ and $\theta_{3}$ can gotten..

\subsection{Forwardkinematics analysis of the master-mechanism}

According to $\theta_{1}, \theta_{2}$ and ${ }^{\theta_{3}}$,getting the $\mathrm{x}, \mathrm{y}$ and $\mathrm{z}$ is the forwardkinematics of master-mechanism.

Given the following $\varphi_{1}, \omega_{1}$ and $\rho_{1}$ according toformula (3):

$$
\begin{gathered}
\varphi_{1}=\mathrm{r} \cos \partial_{1}-R \cos \partial_{1}-\mathrm{L}_{1} \cos \partial_{1} \cos \theta_{1}, \\
\omega_{1}=\mathrm{r} \sin \partial_{1}-R \sin \partial_{1}-\mathrm{L}_{1} \sin \partial_{1} \cos \theta_{1}, \\
\rho_{1}=-\mathrm{L}_{1} \sin \theta_{1}, \\
\left(\mathrm{x}+\varphi_{1}\right)^{2}+\left(\mathrm{y}+\omega_{1}\right)^{2}+\left(\mathrm{z}+\rho_{1}\right)^{2}=L_{2}{ }^{2}
\end{gathered}
$$

Similarly,

$$
\begin{aligned}
& \left(\mathrm{x}+\varphi_{2}\right)^{2}+\left(\mathrm{y}+\omega_{2}\right)^{2}+\left(\mathrm{z}+\rho_{2}\right)^{2}=L_{2}{ }^{2} \\
& \left(\mathrm{x}+\varphi_{3}\right)^{2}+\left(\mathrm{y}+\omega_{3}\right)^{2}+\left(\mathrm{z}+\rho_{3}\right)^{2}=L_{2}^{2}
\end{aligned}
$$

The equations can be gotten according to (5), (6) and (7), the result ofthe forwardkinematics of can be calculated using the equations.

\subsection{D simulation of the master-mechanism}

OpenGL is a kind of packet interface that dependent of software and hardware, and can run on multiple operating system.OpenGL is designed according to the principle of computer graphics, and with strict and excellent 3D graphics standard.It has powerful ability of 3D design, and animation and simulation run the effect of realistic and smooth by it. It is suitable for robotsimulation research.

According to the motion characteristics of Delta parallel master-mechanism and each component shape, the 3D simulation of Delta parallel master-mechanism is following Fig.2.

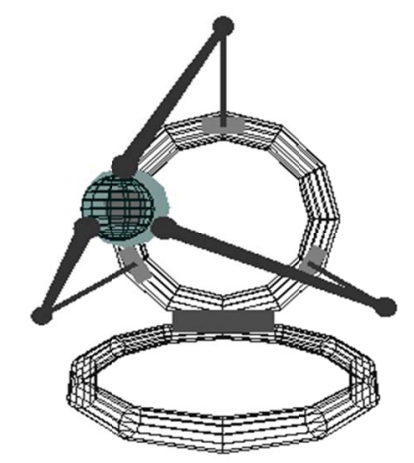

Fig.2:3D simulation of Delta parallel master-mechanism

The basic parameters for the Delta parallel master-mechanism as shown in Table 1.

Table 1:The basic parameters for the Delta parallel master-mechanism

\begin{tabular}{lllll}
\hline & $R$ & $r$ & $L_{1}$ & $L_{2}$ \\
\hline length $(\mathrm{cm})$ & 20 & 4 & 20 & 50 \\
\hline
\end{tabular}

\section{Thekinematic and $3 \mathrm{~d}$ simulation of the slave robot}

Two kinds of structure of robot are designed in the simulation system, but becausethe slaveDeltaparallel robot and Delta master-mechanism have the same structure, kinematics of the SCARA serial robotis analysedin the following, and the structure of kinematics modeling forthe SCARAserial robot is following Fig.3. 


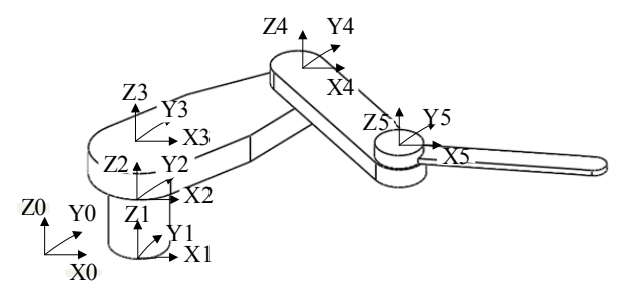

Fig.3:The structure of kinematics modeling fortheslaveSCARAserial robot

\subsection{Forwardkinematics analysis ofthe slaveSCARAserial robot}

Because of the SCARA robot is a serial mechanism, the traditional D-H method is used for model and analysis of kinematics. According to the kinematicsmodelingin Figure 3 , the D-H parameterisobtained as shownin the followingTable 2 .

Table 2:The D-H parameters of SCARA robot

\begin{tabular}{c|c|c|c|c}
\hline $\mathrm{i}$ & $\mathrm{a}_{i-1}$ & $a_{i-1}$ & $d_{i}$ & $\theta_{i}$ \\
\hline 1 & 0 & 0 & 0 & $\theta_{1}$ \\
\hline 2 & 0 & 0 & $d$ & 0 \\
\hline 3 & 0 & 0 & $d_{1}$ & $\theta_{2}$ \\
\hline 4 & 0 & $L_{4}$ & $d_{2}$ & $\theta_{3}$ \\
\hline 5 & 0 & $L_{5}$ & $d_{3}$ & $\theta_{4}$ \\
\hline
\end{tabular}

In order to simplify the calculation, make $L_{4}=L_{5}=L$.According to the table of D-H parametersabove, $T_{5}^{0}$ can be obtained in the following.

$$
T_{5}^{0}=T_{1}^{0} T_{2}^{1} T_{3}^{2} T_{4}^{3} T_{5}^{4}=\left(\begin{array}{cccc}
\cos \left(\theta_{1}+\theta_{2}+\theta_{3}+\theta_{4}\right) & -\sin \left(\theta_{1}+\theta_{2}+\theta_{3}+\theta_{4}\right) & 0 & L^{*}\left(\cos \left(\theta_{1}+\theta_{2}+\theta_{3}\right)+\cos \left(\theta_{1}+\theta_{2}\right)\right) \\
\sin \left(\theta_{1}+\theta_{2}+\theta_{3}+\theta_{4}\right) & \cos \left(\theta_{1}+\theta_{2}+\theta_{3}+\theta_{4}\right) & 0 & L^{*}\left(\sin \left(\theta_{1}+\theta_{2}+\theta_{3}\right)+\sin \left(\theta_{1}+\theta_{2}\right)\right) \\
0 & 0 & 1 & d+d_{2}+d_{3}+d_{4} \\
0 & 0 & 0 & 1
\end{array}\right)
$$

The forwardkinematics of SCARA robot can be obtained according to (8).

\subsection{Inversekinematics analysis of the slaveSCARAserial robot}

The inversekinematics of SCARA robot is that the robot joint angle is obtainedaccording to the position and posture of robot. Because of the SCARA robot is serial mechanism,so that the solution of the inverse kinematics of SCARA robot can use the traditional method of separation of variables.

The solution results are as follows.

$$
\begin{aligned}
& \theta_{1}= \begin{cases}-\arctan \frac{p_{x}}{p_{y}}, & p_{y}>=0 \\
180-\arctan \frac{p_{x}}{p_{y}}, & p_{x}<=0, p_{y}<0 \\
-180-\arctan \frac{p_{x}}{p_{y}}, & p_{x}>0, p_{y}<0\end{cases} \\
& \theta_{2}= \pm 1 / 2 \arccos \frac{2 L^{2}-P_{X}^{2}-P_{Y}^{2}}{2 L^{2}} \\
& \theta_{3}=180-2 \theta_{2} \\
& \theta_{4}=\theta_{2}-90
\end{aligned}
$$

\subsection{D simulation of the slave robot}

According to the motion characteristics of Delta parallel robot andSCARAserial robot, the 3D simulation of the two kinds of robot is following Fig.4 and Fig.5. 


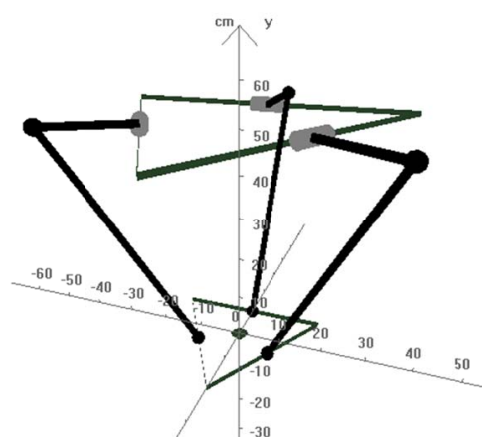

Fig.4:3D simulation of Delta robot

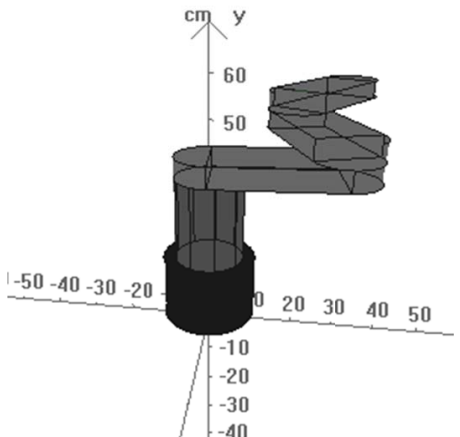

Fig.5:3D simulation of SCARA robot

The basic parameters for theslaveDeltaparallel robot as shown in Table 3

Table 3:The basic parameters for the Delta robot

\begin{tabular}{lllll}
\hline & $\mathrm{R}$ & $\mathrm{r}$ & $L_{1}$ & $L_{2}$ \\
\hline length $(\mathrm{cm})$ & 20 & 4 & 20 & 50
\end{tabular}

The basic parameters for the slaveSCARAserial robot as shown in Table 4

Table 4:The basic parameters for the SCARArobot

\begin{tabular}{llllll}
\hline & $d_{1}$ & $d_{2}$ & $d_{3}$ & $L_{4}$ & $L_{5}$ \\
\hline length $(\mathrm{cm})$ & 5 & 5 & 5 & 30 & 30 \\
\hline
\end{tabular}

\section{TheCommunication platformof3d robot teleoperation simulation system}

In the robot teleoperationcontrol system, communication platform with correctness and stability of data transmission is one of several most important points.Because the TCP protocol is a connection oriented, reliable transmission protocol, it has the characteristics of retransmission for the packet loss, out of order processing and data flow control, by which can guarantee reliability data transmission between the master and the slave. Andthe system uses TCP/IP as the communication protocol of the master and the slave.

Meanwhile, the robot teleoperation control system demands data transmission timely, and reduce transmission delay.About the traditional TCP communication, the client initiates a connection, and will shut down the connection immediately after the data is sent. The clientinitiates a new connectionagain when there is data to be sent, so that process of connection and close will be repeated,which is a waste of a lot of time of data transmission.

So the TCP communication protocol based on the long connection is used in the control system, by which the establishment of connection in the client and the server will not be close because of one end of the data transmission. Thus, the heartbeatcheck is required, conduct the real time monitoring of connection between the client and the server, to carry out data transmission control. And the detailed process is following Fig. 6

Fig.6:TCP communication framework based on a long connection 


\section{The control algorithm ofmaster-slave teleoperation}

The master-slave control algorithm is different betweenthe master-slave isomorphism robot and the master-slave isomeric robot in the robot teleoperationcontrol system.In the control system, the control of Delta robot is an isomorphism control, and the traditional"joint - joint" control method is used, by which directly totransferinformation of each joint angle of the master to the slave, and it can meet the control requirements of the master-slave isomorphism teleoperation.

Becauseof the differences in the master-slave structure and degrees of freedom for the control of SCARA robot, the "joint - joint" control method can't be used. Thus, it need adopt the "joint - pose - pose - joint" control method, namely the end pose data of the master is firstcalculatedaccording to each join angle of the master'smovement, and get the end pose data of the slave through the mapping operation of master-slave hand movement space, finallyconvert the end pose information of the slave to each joint angle of the slave numerical setting and control directly the slave movements through the angles data of the slave.The specific process is shown in belowFig.7.

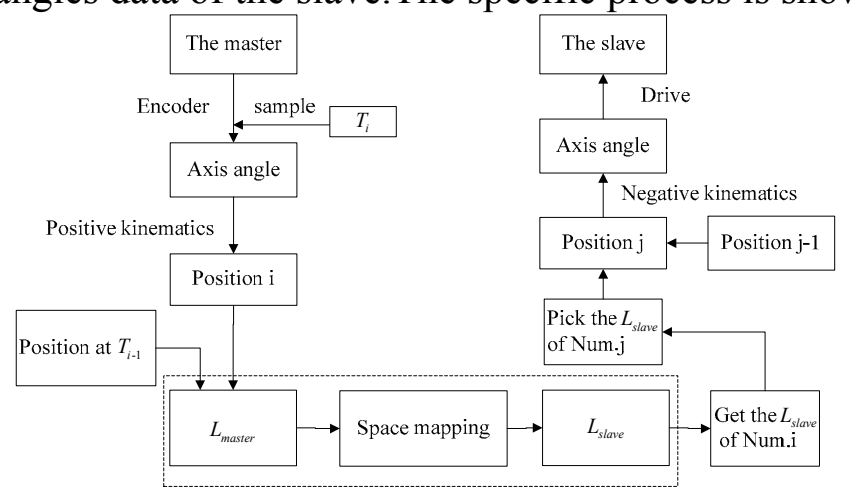

Fig.7:The master-slave control process of the master-slave isomorphism

About the problem of space mappingof master-slave isomeric control, due to the difference in movement space and coordinate system betweenmaster kinematics model and slave kinematics model, so it cannot be directly mapped. The incremental-driven algorithm is taken, namely throughthe positionincrementof the master intwo continuous time, then the position increment in the coordinate system of the slave is gotten through the space mapping method. Thus, the position change of the master can directly map the movement of the slave. The space mappingincludestransformation of the direction and transformation of increment in length.

The coordinate system for robot kinematics model of the master and the slave is shown in the Fig.8.
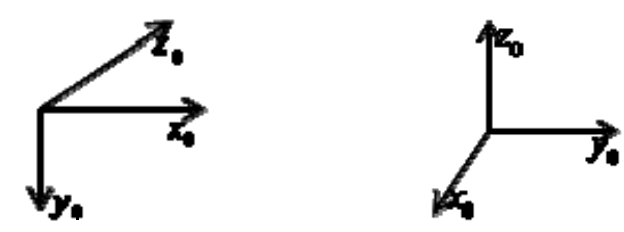

Fig.8:The coordinate system for robot kinematics mode of the master and the slave

Transformation in direction for the mapping relationship is shown in the following Table 5.

Table 5:Transformation in direction for the mapping relationship

\begin{tabular}{|c|c|c|}
\hline Coordinate axis & The master & The slave \\
\hline 1 & $\mathrm{X}$ & $\mathrm{Y}$ \\
\hline 2 & $\mathrm{Y}$ & $-\mathrm{Z}$ \\
\hline 3 & $\mathrm{Z}$ & $-\mathrm{X}$ \\
\hline
\end{tabular}

Transformation of increment in lengthfor the mapping relationship is shown in thebelow. 


$$
k=\frac{L_{\text {master }}}{L_{\text {slave }}} \text {, and }\left\{\begin{array}{l}
K=1, \text { To be consistent in length increment } \\
K>1, \text { To improve the control precision } \\
0<K<1, \text { To expand the operation space }
\end{array}\right.
$$

\section{The design and experiment in robot teleoperation simulation system}

The simulation platform ofrobot teleoperation control system is developedin the Windowssystem.

In view of the simulation platform needs the powerful graphical tools do support, OpenGL as a kind of excellent graphics developmentinterfacecan be closely combined with Windows, so that the simulation platform is developed with the method of mixed programmingcombinedMFC with OpenGLin the Windowssystem.

\subsection{Design of simulation platform ofrobot teleoperation control system}

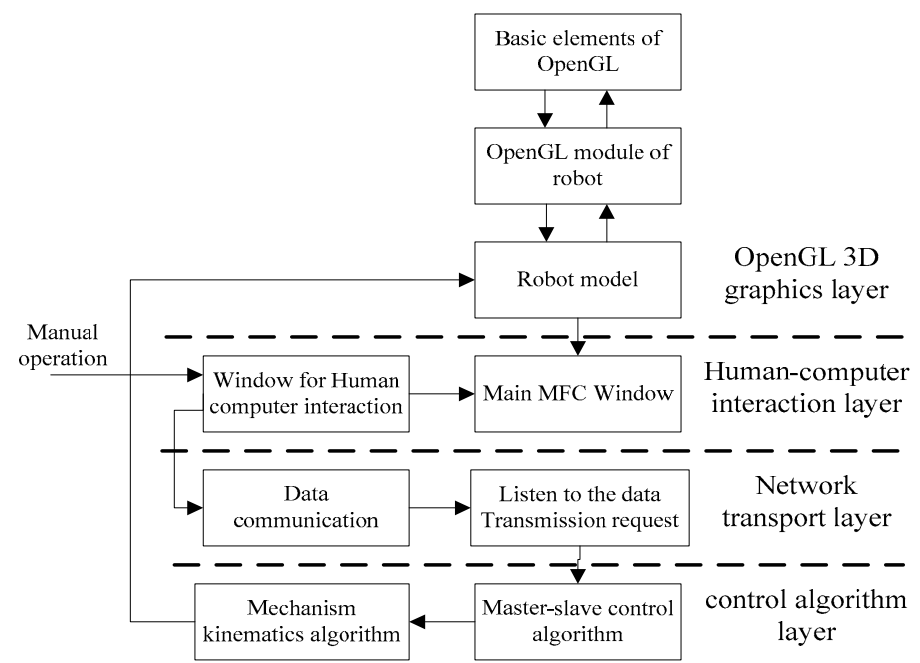

Fig.9:The software architectureof simulation platform

As Figure 9 shows, the simulation platform is composed of software structure included OpenGL 3D graphics layer, human-computer interaction layer, network transport layer and control algorithmlayer.

OpenGL 3D graphics layeris mainly composed of the basic elements drawn by OpenGL ofeach mechanism module of the master or the slave, and then the each mechanismmodule is assembledinto the whole master or the whole slave. The human-computer interaction layer include mainly manual control and state observation based on MFC dialog and OpenGL interactive technology,the network transport layer is used asdata transmission between the master and the slave, and the control algorithm layer mainly include kinematics algorithm of the master or the slave and master-slave control algorithms.

\subsection{The simulation experiment and experiment result}

In order to verify the reliability of the simulation system, the experiment is carried out that the master simulation platform controls the slave simulation platform ofmaster-slaveisomorphism mechanism and the slave simulation platform master-slavealgorithms mechanism. Shown in Figure 10 and Figure 11,master-slave isomorphism robot and the master-slaveisomeric robot are controlledrespectively by the master. Through the analysis of the control data of the master, the operation data of the slave and the motion of simulation model, the stability of the whole logic architecture and the reliability of algorithm of the simulation system. 


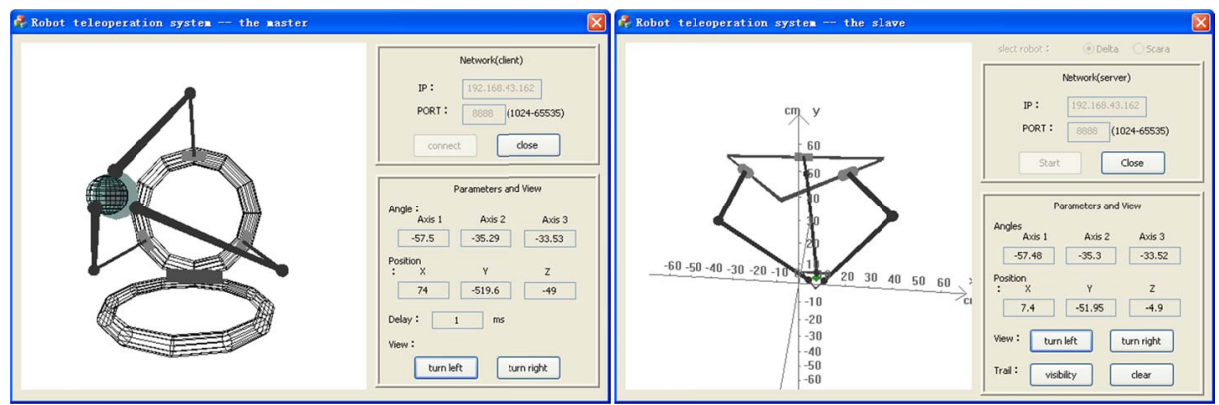

Fig.10:The master controls the slave of master-slaveisomorphism mechanism

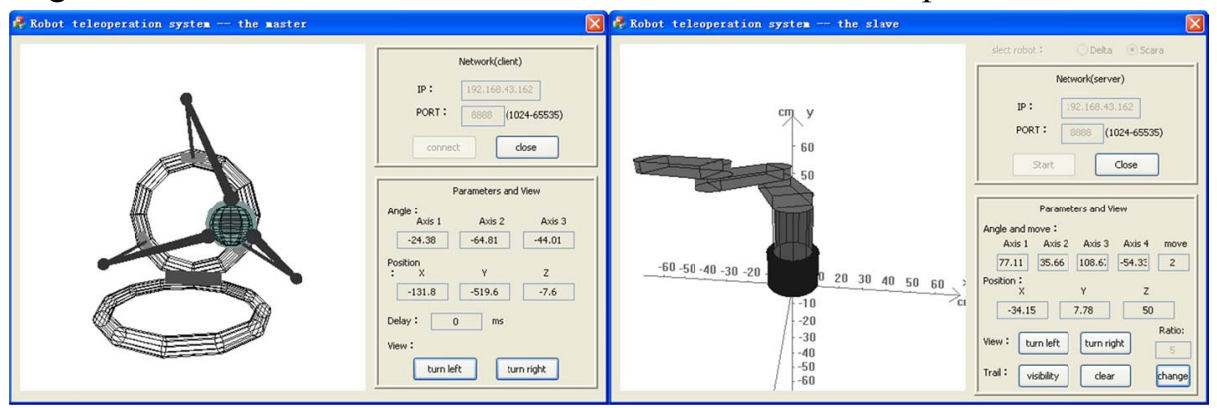

Fig.11:The master controls the slave of master-slaveisomeric mechanism

\section{Conclusion}

In the analysis ofkinematics algorithm of the master and the slave, the 3D simulation model of the master and the slave are established, a long-time connection mode of TCP'communication model is designed,the master-slave control strategy is studied,and the robot teleoperation simulation system is constructed. The simulation results verify the reliabilityof the robot control algorithms and the master-slave control strategy, and the stability of the teleoperation simulation platform. Thus, simulation system achieved the aim for verification and analysis of controlalgorithms of robot teleoperation. The simulation system is easy to operate, the motion data and motion state can be shown in the simulation system. As atool of verificationstrategy and algorithm of robot teleoperation control, it has high research value.

\section{Acknowledgements}

The research work was supported by the National High Technology Research and Development Program ("863" Program)of China 2013AA041005.

\section{References}

[1] Ma Liang, Yan Jihong, Zhao Jie, Chen Zhifeng.Virtual Environment-Based Multi-Operator Multi-Robot Cooperative Teleoperation System[J].Robot,2011,02:169-173.

[2] Shafiqul Islam,Xiaoping P.Liu,Abdulmotaleb El Saddik,LakmalSeneviratne,Jorge Dias. Control Schemes for Passive Teleoperation Systems over Wide Area Communication Networks with Time Varying Delay[J]. International Journal of Automation and Computing,2014,01:100108.

[3] Tang Yusong, Liu Jingtai,Lu Guizhang. Analysis of teleroboticcontrollingsystem based on remote networktechnology[J].Robot,2000,01:67-72+80.

[4] Stapornchaisit, S.; Mitsantisuk, C.; Srisonphan, S.; Chayopitak, N.; Koike, Y.Micro-macro bilateral in task space for delta robot by using forward and inverse kinematic. TENCON $2014-$ 2014 IEEE Region 10 Conference, vol., no., pp.1,6, 22-25 Oct. 2014.

[5] Angel L, Bermudez J, Munoz O. Dynamic optimization and building of a parallel delta-type robot $[\mathrm{C}]$. Proceedings of the Robotics and Biomimetics (ROBIO), 2013 IEEE International Conference on, IEEE, 2013: 444-9. 
[6] Chen Wei, Chen Yimin, Wang, ChenMing. Research on virtual simulation and control of robot [J]. Computer Engineering and Design, 2010,20:4455-4458+4496. 